\title{
Exploiting Lipid Permutation Symmetry to Compute Membrane Remodeling Free Energies
}

\author{
Greg Bubnis, ${ }^{1, *}$ Herre Jelger Risselada, ${ }^{1,2,3}$ and Helmut Grubmüller ${ }^{1, \uparrow}$ \\ ${ }^{1}$ Department of Theoretical and Computational Biophysics, Max-Planck-Institute for Biophysical Chemistry, \\ Göttingen 37077, Germany \\ ${ }^{2}$ Chemistry Department, Leibniz Institute of Surface Modification, Leipzig 04318, Germany \\ ${ }^{3}$ Deptartment of Theoretical Physics, Georg-August University Göttingen, Göttingen 37077, Germany \\ (Received 29 April 2016; revised manuscript received 29 August 2016; published 28 October 2016)
}

\begin{abstract}
A complete physical description of membrane remodeling processes, such as fusion or fission, requires knowledge of the underlying free energy landscapes, particularly in barrier regions involving collective shape changes, topological transitions, and high curvature, where Canham-Helfrich $(\mathrm{CH})$ continuum descriptions may fail. To calculate these free energies using atomistic simulations, one must address not only the sampling problem due to high free energy barriers, but also an orthogonal sampling problem of combinatorial complexity stemming from the permutation symmetry of identical lipids. Here, we solve the combinatorial problem with a permutation reduction scheme to map a structural ensemble into a compact, nondegenerate subregion of configuration space, thereby permitting straightforward free energy calculations via umbrella sampling. We applied this approach, using a coarse-grained lipid model, to test the $\mathrm{CH}$ description of bending and found sharp increases in the bending modulus for curvature radii below $10 \mathrm{~nm}$. These deviations suggest that an anharmonic bending term may be required for $\mathrm{CH}$ models to give quantitative energetics of highly curved states.
\end{abstract}

DOI: 10.1103/PhysRevLett.117.188102

Membrane remodeling is essential for many cellular transport functions, notably fusion [1] and fission [2]. However, despite continued interest, the underlying lipidic mechanisms and energetics are not fully understood. Atomistic and near-atomistic molecular dynamics (MD) simulations provide sufficient temporal and spatial resolution to probe lipidic mechanisms. However, standard MD fails to reach the high energy barriers $\left(\gg k_{B} T\right)$ which often dictate mechanisms and kinetics. Biasing methods are thus required to address the sampling problem for high energy barriers.

An additional and ubiquitous obstacle, in both describing and biasing lipidic transitions, is the $N$ ! degeneracy of lipid configuration space arising from the permutation symmetry of $N$ identical lipids. The same combinatorial symmetry lies at the heart of the Gibbs paradox [3-5], which is resolved by a $1 / N$ ! scaling of the partition function, to account for physically indistinguishable (degenerate) states.

In contrast to this straightforward analytical correction, this permutation symmetry poses a severe challenge to atomistic simulations and free energy calculations involving lipids: sampling the space of degenerate states (via self-diffusion) shrouds the collective structural changes of interest and precludes the use of lipidic collective coordinate biasing schemes. Accordingly, special purpose methods (that circumvent this hindrance) have been developed to direct lipidic transitions using boundary conditions $[6,7]$, external guiding potentials $[8,9]$, probe particles [10], density biasing $[11,12]$, and single-lipid restraints $[13,14]$.
Here, we describe and apply a rigorous method to directly address this $N$ ! degeneracy, using permutation reduction (PR) $[15,16]$. The method exploits permutation symmetry by remapping structures from the full $N$ ! degenerate configuration space into a nondegenerate, compact subregion, thereby overcoming the notorious sampling problem caused by long range lipid self-diffusion. Because the Hamiltonian of the system is invariant under the permutation, the statistical ensemble of the system is unchanged. Importantly, the rigorous definition of collective coordinates also becomes possible for such diffusive systems, as is essential for umbrella sampling free energy calculations.

Here we use and assess this approach to compute membrane bending free energies. In the well established Canham-Helfrich $(\mathrm{CH})$ continuum model $[17,18]$, which treats a membrane as a thin elastic sheet, the bending free energy of a tensionless membrane is

$$
E=\int\left\{\frac{\kappa}{2}\left(H-H_{0}\right)^{2}+\bar{\kappa} K_{G}\right\} d A,
$$

where $H=\left(1 / R_{1}+1 / R_{2}\right), K_{G}=1 /\left(R_{1} R_{2}\right)$ is the Gaussian curvature, and $R_{1}$ and $R_{2}$ are the principal curvature radii for a midplane surface area element $d A$. The three empirical constants $\kappa, H_{0}$, and $\bar{\kappa}$, are the bending modulus, spontaneous curvature, and Gaussian curvature modulus, respectively. For symmetric lipid compositions, $H_{0}=0$, and for a membrane with a fixed surface topology (or when one principal curvature is zero) the $K_{G}$ term integrates to a constant such that (1) reduces to 


$$
E=\int \frac{\kappa}{2} H^{2} d A=\frac{\kappa}{2} \tilde{H}
$$

implying that bending fully dictates the energetics (and thus mechanisms) of nontopological remodeling.

Determining accurate values for $\kappa$ is nontrivial, and the problem has been addressed from different angles (see [19], and references therein). The most prominent simulation schemes derive $\kappa$ from thermal fluctuation spectra of height [20] or lipid tilt [21]. Moreover, despite the widespread use of $\mathrm{CH}$ models for highly curved structures (e.g., an hourglass-shaped fusion stalk) [22,23], it is unclear whether or not the harmonic approximation of constant $\kappa$ is sufficiently accurate in this regime. The first simulations to test this approximation at higher curvatures $[6,24]$ used heavily coarse-grained lipid models, with conflicting results; however, two recent studies have reported anharmonic behavior for binary [25] and ternary (raft-forming) lipid mixtures [26]. We therefore apply our proposed method to compute bending free energies and $\kappa$ up to very high curvatures to rigorously test the range of validity of the harmonic approximation.

We will proceed in three steps. First, PR will be used to derive a suitable collective bending coordinate from an unbiased nonequilibrium trajectory. Second, using this coordinate, PR will be used in conjunction with umbrella sampling to compute a bending free energy profile. Finally, we will compare our results with $\mathrm{CH}$ theory.

An initial unbending pathway $\mathbf{x}(t)$, in the full dimensional configurational space was derived from an unrestrained molecular dynamics (MD) simulation [27] of a membrane half-cylinder [Fig. 1(a)] that relaxes to a flat sheet. The membrane comprises $N=1066$ dioleoylphosphatidylcholine (DOPC) lipids, modeled with the Martini coarse-grained force field [28]. Simulations used periodic boundary conditions; however, the membrane was periodic only along its cylindrical axis. The line tension energy of the two exposed edges was held constant by constraining the box in the $z$ dimension. (Full simulation details are given in the Supplemental Material [29], which includes Refs. [30,31].) During relaxation, lipids freely traveled around the exposed edges to relieve tension asymmetry, but no flip-flops through the membrane (with barriers of $\approx 30 k_{B} T$ [28]) occurred.

Next, we applied PR to the obtained trajectory $\mathbf{x}(t)$. Here the remapping was applied for each frame at time $t_{j}$ by minimizing the $3 N$ Euclidean distances to a reference $\mathbf{x}^{\text {ref,PR }}[15,16]$. To this end, a cost function

$$
\begin{aligned}
\mathcal{C}(\boldsymbol{\pi}, \mathbf{x})= & \left\|\boldsymbol{\pi} \cdot \mathbf{x}_{\{\alpha\}}\left(t_{j}\right)-\mathbf{x}_{\{\alpha\}}^{\mathrm{ref}, \mathrm{PR}}\right\|^{2} \\
& +\sum_{i \in\{\alpha\}} a \theta_{d^{\prime}}\left(\left|\boldsymbol{\pi} \cdot \mathbf{x}_{i}\left(t_{j}\right)-\boldsymbol{\pi}_{j-1}^{*} \cdot \mathbf{x}_{i}\left(t_{j-1}\right)\right|-d^{\prime}\right)
\end{aligned}
$$
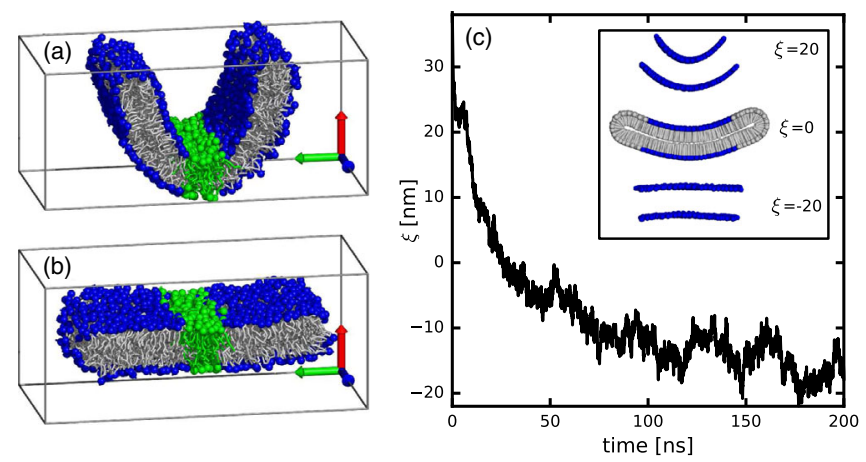

FIG. 1. The permuted DOPC unbending trajectory $\tilde{\mathbf{x}}(t)$ at 0 ns (a) and $80 \mathrm{~ns}$ (b). Solvent is not shown and PO4 beads used for PR are shown as spheres. Lipid headgroups (PO4 and NC3 beads) are colored blue (dark) except for a central patch of green (light) lipids which illustrate how PR preserves lipid sorting throughout the unbending process. The red, green and blue arrows in (a) and (b) indicate the system $x, y$, and $z$ axes, respectively. (c) (main) The PCA derived collective coordinate $\xi$ for $\tilde{\mathbf{x}}(t)$. (inset) The leading PCA mode $\mathbf{v}_{1}$. (inset center) The average permuted structure $\langle\tilde{\mathbf{x}}(t)\rangle$ between 0 and $80 \mathrm{~ns}$. The $650 \mathrm{PO} 4$ beads used for PCA (indices $\{\beta\}$ ) are colored blue (dark) and those not considered are gray (light). The linear mode $\mathbf{v}_{1}$ is illustrated for three cases as $\langle\tilde{\mathbf{x}}(t)\rangle+\xi \cdot \mathbf{v}_{1}$ with $\xi=\{-20,0,20\} \mathrm{nm}$, from bottom to top, respectively.

was minimized using a linear assignment algorithm with $\mathcal{O}\left(N^{3}\right)$ complexity, which yielded the optimal permutation $\boldsymbol{\pi}_{j}^{*}$ of lipid indices. Here, $\theta_{d^{\prime}}(d)$ is a Heaviside step function, $a=500 \mathrm{~nm}^{2}$, frames are at $40 \mathrm{ps}$ intervals, and lipid positions are represented by one bead per lipid (PO4 headgroup beads), indicated by the $\{\alpha\}$ subscripts. The first (compactness) term of (3), is the squared configuration space distance between a permuted labeling of $\mathbf{x}_{\{\alpha\}}\left(t_{j}\right)$, and the reference $\mathbf{x}^{\text {ref,PR }}$ (here, a structure with an intermediate curvature). The second (continuity) term in (3) penalizes single-lipid displacements $d>d^{\prime}$ such as swaps of lipid molecules between the two leaflets, which give rise to discontinuities in the trajectory. (Here, $d^{\prime}=1.4 \mathrm{~nm}$ was used.) The subsequent results were insensitive to the particular choice of $\mathbf{x}^{\text {ref,PR }}$ and $d^{\prime}$ (details are given in the Supplemental Material [29]).

This permutation transformation enabled us to derive via principal component analysis (PCA) a linear order parameter $\xi$ for subsequent umbrella sampling free energy calculations [32]. Here, $\xi[\tilde{\mathbf{x}}(t)]=\left(\tilde{\mathbf{x}}_{\{\beta\}}(t)-\left\langle\tilde{\mathbf{x}}_{\{\beta\}}\right\rangle\right) \cdot \mathbf{v}_{1}$, with $\mathbf{v}_{1}$ being the eigenvector with largest eigenvalue of the $3 N_{\beta} \times 3 N_{\beta}$ covariance matrix calculated from the trajectory $\tilde{\mathbf{x}}(t)$. To avoid boundary artifacts, only the 650 centermost PO4 beads were considered to define $\xi$, indicated by subscripts $\beta$. As can be seen in Fig. 1, by construction this projection defines $\xi$ using the collective coordinate which describes the largest collective atomic motion for the membrane deformation. Moreover, because $\xi$ is linear, the applied umbrella potential 
$V_{\text {umb }}(\tilde{\mathbf{x}})=(k / 2)\left[\xi\left(\tilde{\mathbf{x}}_{\{\beta\}}\right)-\xi\left(\tilde{\mathbf{x}}_{\{\beta\}}^{\text {ref }}\right)\right]^{2}$, where $k$ is the force constant, restrains only one (collective) degree of freedom in the system and, in this sense, introduces the smallest possible bias towards a particular reaction coordinate.

Crucially, $V_{\text {umb }}(\tilde{\mathbf{x}})$ takes structures that have been permuted by the same PR rule used to derive $\tilde{\mathbf{x}}(t)$ and $\xi$. To fulfill this requirement, and to prevent the system from escaping the restraints by lateral diffusion perpendicular to $\mathbf{v}_{1}$, PR was applied at $40 \mathrm{ps}$ intervals during the umbrella sampling MD simulations. These PR corrections cause small discontinuities in configuration space with resulting jumps in the potential energy of $\sigma_{V_{\mathrm{umb}}}<1 \mathrm{~kJ} / \mathrm{mol}$. (see Supplemental Material [29] for details).

The umbrella ensemble comprised 16 windows (indices $l$ ) taking their reference and initial structures $\tilde{\mathbf{x}}^{\text {ref, } l}$ from $\tilde{\mathbf{x}}(t)$, spaced approximately evenly in $\xi$ between -22 and $38 \mathrm{~nm}$. All umbrellas used $\left.k=1.0 \mathrm{~kJ} /(\mathrm{mol} \mathrm{nm})^{2}\right)$. During MD simulations, PR used the unbending average $\langle\tilde{\mathbf{x}}(t)\rangle$ [shown in Fig. 1(c)(inset)(center)] as $\mathbf{x}^{\mathrm{ref}, \mathrm{PR}}$ and $d^{\prime}=$ $1.4 \mathrm{~nm}$ (for details of the simulation protocol, see the Supplemental Material [29]).

Following $50 \mathrm{~ns}$ of equilibration and $450 \mathrm{~ns}$ of production MD per window, we computed the potential of mean force (PMF) $E(\xi)$ [see Fig. 2(a)] using the weighted histogram analysis method (WHAM) [33,34]. The resulting small statistical uncertainty of the PMF $\left(\sigma_{E}<2 k_{B} T\right)$ and the short autocorrelation times for $\xi(10-20 \mathrm{~ns})$ indicate that sufficient sampling was obtained across the studied range of curvature and illustrate the utility of this approach for precise free energy calculations of biologically relevant high energy and curvature states. The most highly curved membranes adopted parabolic rather than cylindrical shapes [e.g., structure 2 of Fig. 2(a)], with $H$ being highest at the center. We ascribe this effect to the $H=0$ (on average) boundary conditions imposed by the free edges [gray lipid headgroups in Fig. 1(c)(inset)]. Subsequent curvature calculations, detailed in the Supplemental Material [29], account for these noncylindrical shapes.

For comparison with the $\mathrm{CH}$ description of bending, $E(\xi)$ was recast according to (2), as $E(\tilde{H})$, a function of midplane curvature. Figure 2(b) shows that $E(\tilde{H})$ (black points, green quadratic fit) is nonlinear, indicating that the membrane stiffens with increasing curvature, and that the harmonic approximation (linear, red component) indeed becomes inaccurate at high curvature.

This nonlinearity is accounted for by adding a quartic bending term $(\mu / 4) H^{4}$ to the integrand of (2) [6,26,35-37] and integrating to obtain a sum of quadratic and quartic energy terms,

$$
E=E_{2}+E_{4}=\frac{\kappa}{2} H_{2}+\frac{\mu}{4} H_{4},
$$

where $H_{2}=\tilde{H}=\int H^{2} d A$ and $H_{4}=\int H^{4} d A$. (The associated quartic $K_{G}$ terms $[35,38,39]$ are safely neglected
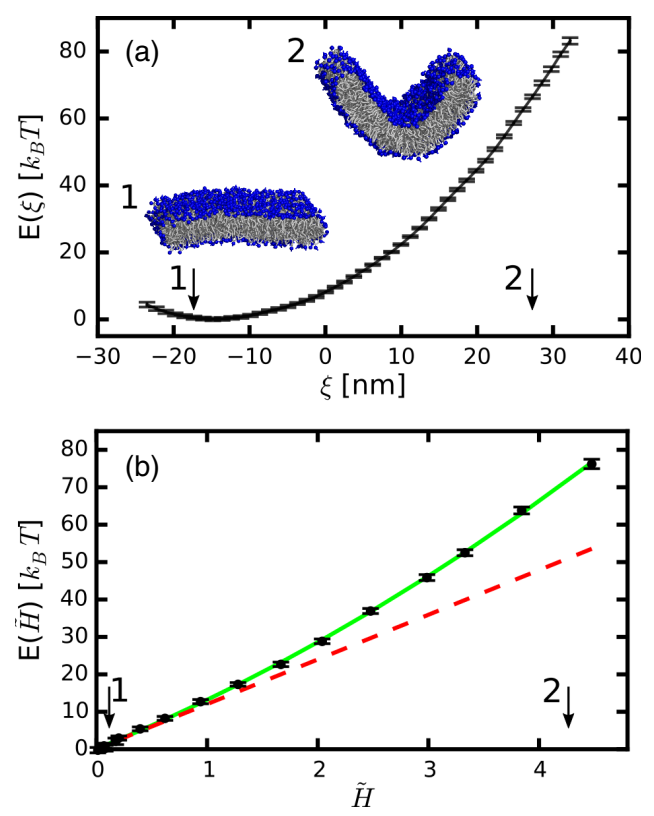

FIG. 2. (a) The bending free energy (PMF) $E(\xi)$ for DOPC from $16 \times 450 \mathrm{~ns}$ umbrella sampling simulations. $E(\xi)$ was recovered using WHAM and the error bars are the standard deviations of 30 bootstrapped PMFs. (b) The bending free energy $E(\tilde{H})$ as a function of the Helfrich curvature integral $\tilde{H}$. Points lie at the umbrella window means $\langle\tilde{H}\rangle$ and error bars are $95 \%$ confidence intervals. The quadratic fit is shown in green (solid line), and its linear component, the harmonic Helfrich approximation, in red (dashed line). Two snapshots (1 and 2) in (a) depict the range of bending in the ensemble and arrows in (a) and (b) show the $\xi$ and $\tilde{H}$ values. For structure $2, R=4.6 \mathrm{~nm}$ at its center.

because $K_{G}=0$.) In Fig. 2(b), the linear component $E_{2}$ yields $\kappa$, and the excess nonlinear energy $E_{4}$ gives the quartic modulus $\mu$. [For details of the change of coordinates $\xi \rightarrow\left(H_{2}, H_{4}\right)$ and of computing $\kappa$ and $\mu$, see the Supplemental Material [29].] Finally, from $\kappa$ and $\mu$, a curvature dependent, effective bending modulus can be defined as $\kappa_{\text {eff }}=\kappa+(\mu / 2) H^{2}$ or, equivalently, $\kappa_{\text {eff }}=$ $\kappa\left(1+\left[R_{\mu} H\right]^{2}\right)$, where the distance $R_{\mu}=\sqrt{\mu / 2 \kappa}$ indicates the length scale where $\kappa$ increases $[6,26]$.

Next, we asked how $\kappa_{\text {eff }}$ depends on tail saturation, tail length, and headgroup polarity. Figure 3 and Table I compare $\kappa, \mu$, and $R_{\mu}$ for pure dioleoylphosphatidylethanolamine (DOPE), distearoylphosphatidylcholine (DSPC), dipalmitoylphosphatidylcholine (DPPC), and dimyristoylphosphatidylcholine (DMPC) membranes, all in the liquid disordered $L_{\alpha}$ phase $[28,40]$.

At low curvatures, the saturated lipids DMPC, DPPC, and DSPC showed $\kappa$ increases of about $8 k_{B} T$ for each increase in tail length (one bead represents approximately 4 carbons); this trend is known from micropipette aspiration experiments [41] and continuum mechanics [42]. Also, the decrease of $\kappa$ from DSPC to DOPC is in line with aspiration experiments showing that unsaturated alkyl chains decrease 


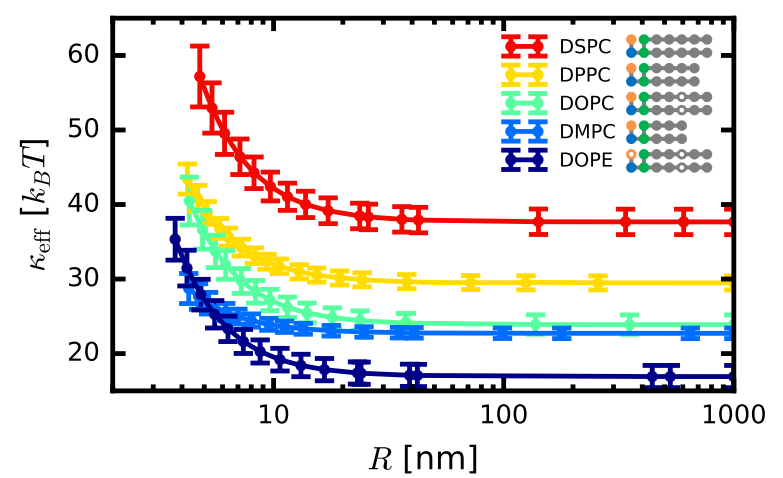

FIG. 3. Midplane (curvature) radius dependent bending moduli, $\kappa_{\text {eff }}(R)$. Points lie at the mean minimum curvature radius $\left\langle R_{\min }\right\rangle$ of each umbrella window. Error bars are $95 \%$ confidence intervals. Note that $\kappa_{\text {eff }}(R)=\kappa$ at large $R$. The legend depicts Martini lipids and their constituent coarse-grained beads. The bead types are: gray solid or open; saturated or unsaturated carbon chain (approximately 4 carbons per bead), green; glycerol, blue; PO4, orange solid or open; NC3 or NH3 (choline or amine).

$\kappa$ [41]. Because DOPE is in the inverse hexagonal $\left(H_{\mathrm{II}}\right)$ phase at $310 \mathrm{~K}$ [43], whereas DOPC forms $\left(L_{\alpha}\right)$ bilayers, no direct comparison of their elastic properties is available. However, the smaller $\kappa$ seen in our simulations for DOPE suggests that PE headgroup dehydration at the upper (negative intrinsic curvature) leaflet is more favorable than for DOPC, consistent with the smaller hydration shells of the PE headgroups, which imply smaller hydration repulsion. At low curvature, our $\kappa$ results also agree with previous simulation results $[7,21,44-52]$ (a table is presented in the Supplemental Material [29]).

At high curvature, when $R$ is ca. twice the membrane thickness, $\kappa_{\text {eff }}$ increases for all five lipids (see Fig. 3), most prominently for DOPC, DOPE, and DSPC (all have 14 beads per lipid); however, the effect decreases for shorter tails, as the saturated PC series (DSPC, DPPC, DMPC) shows. For DMPC the effect is relatively small. The $R_{\mu}$ values in Table I also corroborate the location and strength of the anharmonic increases in $\kappa_{\text {eff }}$. The differences in $R_{\mu}$ between DOPC, DOPE, and DSPC are too small to suggest that headgroup polarity or chain saturation significantly alter the anharmonicity. But together, these trends suggest that the observed anharmonicity is chiefly a function of lipid tail length. This onset of stiffening at $R \lesssim 10 \mathrm{~nm}$ also qualitatively agrees with recent results obtained by

TABLE I. Computed values of $\kappa, \mu$, and $R_{\mu}$, all at $T=310 \mathrm{~K}$.

\begin{tabular}{lclc}
\hline \hline Lipid & $\kappa\left[k_{B} T\right]$ & $\mu\left[k_{B} T \mathrm{~nm}^{2}\right]$ & $R_{\mu}[\mathrm{nm}]$ \\
\hline DOPC & $23.9 \pm 1.3$ & $614 \pm 109$ & $3.59 \pm 0.32$ \\
DOPE & $16.9 \pm 1.5$ & $514 \pm 66$ & $3.89 \pm 0.27$ \\
DSPC & $37.7 \pm 1.7$ & $891 \pm 169$ & $3.44 \pm 0.33$ \\
DPPC & $29.5 \pm 0.9$ & $498 \pm 64$ & $2.91 \pm 0.18$ \\
DMPC & $22.7 \pm 0.7$ & $220 \pm 70$ & $2.20 \pm 0.35$ \\
\hline \hline
\end{tabular}

Vidal et al., who simulated a 1:1 (Martini) DPPC/DOPE mixture [25].

To validate our PR approach, we used two additional methods to extract $\kappa$ at the curvature extremes (for DOPC): cylindrical tethers (CT) [6,25], with radii $R$ from 4.6$8.6 \mathrm{~nm}$, and height fluctuation (HF) spectroscopy for a flat periodic sheet $\left(R \approx 200 \mathrm{~nm}\right.$ for $\left.q=0.16 \mathrm{~nm}^{-1}\right)$ [20]. These methods gave $\kappa^{\mathrm{CT}}=42.1 \pm 0.9$ (at $R=4.6 \mathrm{~nm}$ ) and $\kappa^{\mathrm{HF}}=30.6 \pm 1.9 k_{B} T$, respectively, confirming the anharmonic trend of Fig. 3. For $R<7 \mathrm{~nm}, \kappa^{\mathrm{CT}}$ values were larger than $\kappa_{\mathrm{eff}}^{\mathrm{PR}}$ by about $5 k_{B} T$, slightly outside our estimated uncertainties. In the weak curvature regime, $\kappa^{\mathrm{HF}}$ was larger than $\kappa_{\mathrm{eff}}^{\mathrm{PR}}$ by about $7 k_{B} T$; however, HF is known to overestimate $\kappa$ (see [49]) for small and midsized membranes that cannot probe sufficiently large wavelengths (small $q$ ). In contrast, a recent field theoretical simulation scheme gave $\kappa=19 \pm 4 k_{B} T$ [52], which is 5 $k_{B} T$ less than $23.9 k_{B} T$ computed here, further suggesting that $\kappa^{\mathrm{HF}}$ is an overestimate. Finally, we verified that $\kappa_{\text {eff }}$ was independent of the particular choice of parameters $d^{\prime}$ and $k$ as well as the definition of $\xi$. In only one case, using a single leaflet $\xi, \kappa$, and $\mu$ increased by $18 \%$ and $76 \%$, respectively. For full descriptions of the CT/HF results and controls, see the Supplemental Material [29].

Two lines of reasoning indicate that the physical, microscopic origin of increasing $\kappa_{\text {eff }}$ involves frustrated tail packing of the positive (intrinsic) curved leaflet. First, the absence of this effect in $H_{\text {II }}$ monolayers [53] suggests that any $\kappa_{\text {eff }}$ increase should arise from positive curvature. Second, the CT simulations in [6], using a soft, highly coarse-grained lipid model showed no evidence of anharmonicity, suggesting that the effect might originate from stronger repulsions in the more physical model used here. Such an imbalance between tail repulsion and headgroup attraction, emerging at high curvature, would depend sensitively on the position of the pivotal plane [54] and should also increase with bilayer thickness.

To summarize, in this Letter we have applied a permutation symmetry reduction scheme to lipid membranes, rendering the biologically relevant, high curvature regime accessible to umbrella sampling free energy calculations. We have applied this approach to test the harmonic bending approximation of the $\mathrm{CH}$ model and observed a sharp increase in the bending modulus $\kappa$ at curvature radii below $10 \mathrm{~nm}$. This breakdown of the harmonic approximation suggests that an additional bending term (and its corresponding modulus $\mu$ ) is required for the $\mathrm{CH}$ model to give quantitative energetics of highly curved membranes. Moreover, these refinements are essential for $\mathrm{CH}$ models to predict kinetics, which are exponentially sensitive to barrier heights. Beyond the present study of bending, many topological processes (poration, fusion stalk formation) remain both poorly understood and inaccessible to $\mathrm{CH}$ descriptions, but should be accessible to PR based free energy calculations. More generally, PR could help elucidate collective phenomena for other classes of soft or interfacial 
systems that possess permutation symmetry including colloids, nanoparticles, thin films, and (bio)polymers.

The authors gratefully acknowledge helpful discussions with Jochen Hub, Neha Awasthi, Marcus Müller, and Yuliya Smirnova as well as suggestions from an anonymous reviewer that substantially improved this work. Financial support from the Deutsche Forschungsgemeinschaft (DFG) Grant No. SFB 803.B2 is also gratefully acknowledged.

*gbubnis@gwdg.de

†hgrubmu@gwdg.de

[1] R. Jahn and D. Fasshauer, Molecular machines governing exocytosis of synaptic vesicles, Nature (London) 490, 201 (2012).

[2] S. Morlot and A. Roux, Mechanics of dynamin-mediated membrane fission, Annu. Rev. Biophys. 42, 629 (2013).

[3] J. W. Gibbs, in The Scientific Papers of J. Willard Gibbs (Dover Publications, Inc., New York, 1961), p. 1875.

[4] J. W. Gibbs, in The Collected Works of J. Willard Gibbs (Dover Publications, Inc., New York, 1960), Vol. 2, p. 1902.

[5] E. T. Jaynes, in Maximum Entropy and Bayesian Methods (Kluwer Academic Publishers, Dordrecht, 1992).

[6] V. A. Harmandaris and M. Deserno, A novel method for measuring the bending rigidity of model lipid membranes by simulating tethers, J. Chem. Phys. 125, 204905 (2006).

[7] M. Hu, P. Diggins, and M. Deserno, Determining the bending modulus of a lipid membrane by simulating buckling, J. Chem. Phys. 138, 214110 (2013).

[8] S. Kawamoto, T. Nakamura, S. O. Nielsen, and W. Shinoda, A guiding potential method for evaluating the bending rigidity of tensionless lipid membranes from molecular simulation, J. Chem. Phys. 139, 034108 (2013).

[9] W. Shinoda, T. Nakamura, and S. O. Nielsen, Free energy analysis of vesicle-to-bicelle transformation, Soft Matter 7, 9012 (2011).

[10] H. J. Risselada, G. Bubnis, and H. Grubmuller, Expansion of the fusion stalk and its implication for biological membrane fusion, Proc. Natl. Acad. Sci. U.S.A. 111, 11043 (2014).

[11] T. V. Tolpekina, W. K. den Otter, and W. J. Briels, Nucleation free energy of pore formation in an amphiphilic bilayer studied by molecular dynamics simulations, J. Chem. Phys. 121, 12060 (2004).

[12] M. Muller, Y. G. Smirnova, G. Marelli, M. Fuhrmans, and A.-C. Shi, Transition Path from Two Apposed Membranes to a Stalk Obtained by a Combination of Particle Simulations and String Method, Phys. Rev. Lett. 108, 228103 (2012).

[13] Y. G. Smirnova, S.-J. Marrink, R. Lipowsky, and V. Knecht, Solvent-exposed tails as prestalk transition states for membrane fusion at low hydration, J. Am. Chem. Soc. 132, 6710 (2010).

[14] W. F. Drew Bennett and D. Peter Tieleman, Water defect and fore formation in atomistic and coarse-grained lipid membranes: Pushing the limits of coarse graining, J. Chem. Theory Comput. 7, 2981 (2011).
[15] F. Reinhard and H. Grubmuller, Estimation of absolute solvent and solvation shell entropies via permutation reduction, J. Chem. Phys. 126, 014102 (2007).

[16] F. Reinhard, O. F. Lange, J. S. Hub, J. Haas, and H. Grubmuller, gpermute: Permutation-reduced phase space density compaction, Comput. Phys. Commun. 180, 455 (2009).

[17] P. B. Canham, The minimum energy of bending as a possible explanation of the biconcave shape of the human red blood cell, J. Theor. Biol. 26, 61 (1970).

[18] W. Helfrich, Elastic properties of lipid bilayers: Theory and possible experiments, Z. Naturforsch. C 28, 693 (1973).

[19] J. F. Nagle, Introductory lecture: Basic quantities in model biomembranes, Faraday Discuss. 161, 11 (2013).

[20] R. Goetz, G. Gompper, and R. Lipowsky, Mobility and Elasticity of Self-Assembled Membranes, Phys. Rev. Lett. 82, 221 (1999).

[21] M. C. Watson, E. G. Brandt, P. M. Welch, and F. L. H. Brown, Determining Biomembrane Bending Rigidities from Simulations of Modest Size, Phys. Rev. Lett. 109, 028102 (2012).

[22] M. M. Kozlov and M. Winterhalter, Elastic moduli for strongly curved monoplayers: Position of the neutral surface, J. Phys. II (France) 1, 1077 (1991).

[23] D. P. Siegel, Energetics of intermediates in membrane fusion: Comparison of stalk and inverted micellar intermediate mechanisms, Biophys. J. 65, 2124 (1993).

[24] W. K. den Otter and W. J. Briels, The bending rigidity of an amphiphilic bilayer from equilibrium and nonequilibrium molecular dynamics, J. Chem. Phys. 118, 4712 (2003).

[25] I. A. Barragán Vidal, C. M. Rosetti, C. Pastorino, and M. Muller, Measuring the composition-curvature coupling in binary lipid membranes by computer simulations, J. Chem. Phys. 141, 194902 (2014).

[26] H. J. Risselada, S. J. Marrink, and M. Muller, CurvatureDependent Elastic Properties of Liquid-Ordered Domains Result in Inverted Domain Sorting on Uniaxially Compressed Vesicles, Phys. Rev. Lett. 106, 148102 (2011).

[27] B. Hess, C. Kutzner, D. van der Spoel, and E. Lindahl, GROMACS 4 algorithms for highly efficient, loadbalanced, and scalable molecular simulation, J. Chem. Theory Comput. 4, 435 (2008).

[28] S. J. Marrink, H. J. Risselada, S. Yefimov, D. Peter Tieleman, and A. H. de Vries, The MARTINI force field: Coarse grained model for biomolecular simulations, J. Phys. Chem. B 111, 7812 (2007).

[29] See Supplemental Material at http://link.aps.org/ supplemental/10.1103/PhysRevLett.117.188102 for simulation methods, curvature energy calculations, an analysis of coupling between permutations and collective coordinates, and control simulations.

[30] W. K. den Otter, Free energies of stable and metastable pores in lipid membranes under tension, J. Chem. Phys. 131, 205101 (2009).

[31] J. D. Chodera, W. C. Swope, J. W. Pitera, C. Seok, and K. A. Dill, Use of the weighted histogram analysis method for the analysis of simulated and parallel tempering simulations, J. Chem. Theory Comput. 3, 26 (2007).

[32] G. M. Torrie and J. P. Valleau, Nonphysical sampling distributions in Monte Carlo free-energy estimation: Umbrella sampling, J. Comput. Phys. 23, 187 (1977). 
[33] J. S. Hub, B. L. de Groot, and D. van der Spoel, gwham: A free weighted histogram analysis implementation including robust error and autocorrelation estimates, J. Chem. Theory Comput. 6, 3713 (2010).

[34] S. Kumar, J. M. Rosenberg, Djamal Bouzida, R. H. Swendsen, and P. A. Kollman, The weighted histogram analysis method for free-energy calculations on biomolecules. I. The method, J. Comput. Chem. 13, 1011 (1992).

[35] M. D. Mitov, Third and fourth order curvature elasticity of lipid bilayers, C.R. Acad. Bulg. Sci. 31, 513 (1978).

[36] M. Deserno, Fluid lipid membranes: From differential geometry to curvature stresses, Chem. Phys. Lipids 185, 11 (2015).

[37] J. B. Fournier and P. Galatola, Tubular vesicles and effective fourth-order membrane elastic theories, Europhys. Lett. 39, 225 (1997).

[38] D. P. Siegel, Determining the ratio of the Gaussian curvature and bending elastic moduli of phospholipids from $Q_{\mathrm{II}}$ phase unit cell dimensions, Biophys. J. 91, 608 (2006).

[39] T. Shemesh, A. Luini, V. Malhotra, K. N. J. Burger, and M. M. Kozlov, Prefission constriction of Golgi tubular carriers driven by local lipid metabolism: A theoretical model, Biophys. J. 85, 3813 (2003).

[40] S. J. Marrink, J. Risselada, and A. E. Mark, Simulation of gel phase formation and melting in lipid bilayers using a coarse grained model, Chem. Phys. Lipids 135, 223 (2005).

[41] W. Rawicz, K. C. Olbrich, T. McIntosh, D. Needham, and E. Evans, Effect of chain length and unsaturation on elasticity of lipid bilayers, Biophys. J. 79, 328 (2000).

[42] L. D. Landau and E. M. Lifshitz, Theory of Elasticity (Butterworth-Heinemann, Oxford, 1999).

[43] K. Gawrisch, V. A. Parsegian, D. A. Hajduk, M. W. Tate, S. M. Gruner, N. L. Fuller, and R. P. Rand, Energetics of a hexagonal-lamellar-hexagonal-phase transition sequence in dioleoylphosphatidylethanolamine membranes, Biochemistry 31, 2856 (1992).
[44] J. Wong-Ekkabut, S. Baoukina, W. Triampo, I.-M. Tang, D. P. Tieleman, and L. Monticelli, Computer simulation study of fullerene translocation through lipid membranes, Nat. Nanotechnol., 3, 363 (2008).

[45] S. Baoukina, S. J. Marrink, and D. Peter Tieleman, Molecular structure of membrane tethers, Biophys. J. 102, 1866 (2012).

[46] S. J. Marrink, A. H. de Vries, and A. E. Mark, Coarse grained model for semiquantitative lipid simulations, J. Phys. Chem. B 108, 750 (2004).

[47] S Shkulipa, Ph.D. thesis, University of Twente, The Netherlands, 2006.

[48] W. K. den Otter and S. A. Shkulipa, Intermonolayer friction and surface shear viscosity of lipid bilayer membranes, Biophys. J. 93, 423 (2007).

[49] M. C. Watson, E. S. Penev, P. M. Welch, and F. L. H. Brown, Thermal fluctuations in shape, thickness, and molecular orientation in lipid bilayers, J. Chem. Phys. 135, 244701 (2011).

[50] E. G. Brandt, A. R. Braun, J. N. Sachs, J. F. Nagle, and O. Edholm, Interpretation of fluctuation spectra in lipid bilayer simulations, Biophys. J. 100, 2104 (2011).

[51] M. Hu, D. H. de Jong, S. J. Marrink, and M. Deserno, Gaussian curvature elasticity determined from global shape transformations and local stress distributions: A comparative study using the MARTINI model, Faraday Discuss. 161, 365 (2013).

[52] Y. G. Smirnova and M. Muller, Calculation of membrane bending rigidity using field-theoretic umbrella sampling, J. Chem. Phys. 143, 243155 (2015).

[53] A. J. Sodt and R. W. Pastor, Bending free energy from simulation: Correspondence of planar and inverse hexagonal lipid phases, Biophys. J. 104, 2202 (2013).

[54] X. Wang and M. Deserno, Determining the pivotal plane of fluid lipid membranes in simulations, J. Chem. Phys. 143, 164109 (2015). 